\title{
La Protección de la Salud de los Trabajadores en España: una Oportunidad para la Participación, la Negociación y el Consenso en el Marco de las Relaciones Laborales ${ }^{1}$
}

\author{
Ana M García* \\ María José López-Jacob \\ Jerónimo Ramón Díaz Gómez
}

ReSUMEN:

La Ley de Prevención de Riesgos Laborales en España (Ley 31/1995) abrió un espacio nuevo para la participación de los trabajadores en materia de prevención de riesgos laborales a través de los delegados de prevención, representantes de los trabajadores en esta materia, y de los Comités de Seguridady Salud, obligatorios en las empresas con más de 50 trabajadores. La investigación internacional ha demostrado el impacto positivo de la participación de los trabajadores en la mejora de las condiciones de trabajo y en la reducción de los problemas de salud laboral en las empresas. En España contamos ya también con datos que permiten describir las actividades de participación en materia de prevención en las empresas, asi como analizar su impacto en la protección de la salud y el bienestar de los trabajadores. El objetivo general de este trabajo es presentar y comentar la evidencia disponible sobre este tema.

* Departamento de Medicina Preventiva y Salud Pública. Facultad de Ciencias Sociales. Universidad de Valencia • anagar@uv.es

1. Texto de la comunicación presentada en el I Congreso Estatal de Centros Universitarios de Relaciones Laborales y Ciencias del Trabajo celebrado en Valencia, septiembre 2013. Comunicación incluida en el Grupo 1: Transformaciones en el modelo de relaciones laborales español. 
PaLABRAS CLAVE:

Salud laboral; participación; efecto sindicato; prevención de riesgos laborales; evidencia científica.

ABSTRACT:

The Law of Prevention of Occupational Risks in Spain (Law 31/1995) opened a new space for the participation of workers in the field of occupational health through the so-called Prevention Delegates, workers' representatives in this field, and the Safety and Health Committees, mandatory in companies with more than 50 workers. International research has demonstrated the positive impact of workers' participation in working conditions and in workers' occupational health. Nowadays, in Spain we have also data describing the activities of participation related to occupational health and safety in the companies, and their impact on the protection of workers' wellbeing and health. The overall aim of this paper is to present and discuss the available evidence on this subject.

KEYWORDS:

Occupational health; participation; union effect; occupational risk prevention; scientific evidence.

\section{UN SOBREVUELO POR LOS ANTECEDENTES}

La protección de la salud y seguridad en el trabajo en España ha atravesado cambios muy sustanciales en las últimas décadas, aunque algunas instituciones principales y algunas peculiaridades del sistema vienen en gran medida heredados de épocas anteriores (Bernabeu, 2000:76-79). La actual Escuela Nacional de Medicina del Trabajo apareció junto con el entonces llamado Instituto de Medicina, Higiene y Seguridad en el Trabajo (1944), germen igualmente del actual Instituto Nacional de Seguridad e Higiene en el Trabajo. En los años 50 surgen las primeras sociedades profesionales de medicina del trabajo (a nivel nacional y también vinculadas a algunas regiones, como Cataluña y Castilla) y se establecen también los Servicios Médicos de Empresa. A pesar de la proximidad en algún momento de las disciplinas "sanitarias" y "técnicas" en relación con la seguridad y salud en el trabajo, lo cierto es que la adscripción del Instituto de Seguridad e Higiene en el Trabajo (1978) al Ministerio de Trabajo y Seguridad Social (administración laboral) marcan el predominio de profesionales provenientes del mundo de la ingeniería y la jurisprudencia, siguiendo la tradición de la veterana Inspección de Trabajo, mientras que la medicina laboral queda adscrita, o más bien diluida, en la administración sanitaria. Las desafortunadas historias de las especialidades de Medicina del Trabajo y Enfermería del Trabajo en 
nuestro país ilustran perfectamente la marginalidad de estos estudios en el ámbito sanitario (García, 2012:40-45).

La entrada de España en la Comunidad Económica Europea en 1986 es decisiva también para el ulterior desarrollo legislativo en materia de salud y seguridad en el trabajo. En muy poco tiempo, partiendo de estructuras y normativas previas bastante limitadas e insuficientes, como los Servicios de Medicina de Empresa (1959) o la Ordenanza General de Seguridad e Higiene en el Trabajo (1971), España se dota de una Ley de Prevención de Riesgos Laborales (Ley 31/1995) y un Reglamento de los Servicios de Prevención (Reglamento 39/1997) decisivos para configurar las actuales prácticas y estructuras en materia de salud y seguridad en el trabajo. A ambas normativas principales le siguen la aprobación de una muy abundante serie de disposiciones que vienen a regular la protección de la salud de los trabajadores en relación con distintas situaciones de riesgo en el trabajo, disposiciones que en su mayoría son derivadas del ordenamiento europeo (Bernabeu, 2000:79-80).

La Ley 31/1995, fundamentalmente, es la transposición al derecho español de la Directiva Europea 89/391/CEE, pero también de otras directivas como la 92/85/CEE, 94/33/CEE y 91/383/CEE, relativas a la protección de la maternidad, de los jóvenes y al tratamiento de las relaciones de trabajo de duración determinada o de empresas de trabajo temporal, respectivamente. Con la Ley 31/1995 se introducen en España cambios sustanciales en la estructura y prácticas de salud y seguridad en el trabajo. A partir de la entrada en vigor de dicha ley, todas las empresas están obligadas a planificar y organizar sus actividades de prevención de forma sistemática. Otro cambio muy relevante es la creación de estructuras de representación de los trabajadores y de consenso en materia de salud y seguridad en el trabajo. Por primera vez en España aparece la figura del delegado de prevención, representante de los trabajadores con funciones específicas en materia de prevención de riesgos en el trabajo, y se organizan Comités de Seguridad y Salud como estructuras de participación y consenso en las empresas con 50 trabajadores o más. La insistencia en los derechos de participación de los trabajadores en todos los aspectos relacionados con la protección de la salud y seguridad en el trabajo es una constante en toda la normativa española.

Con el paso de los años, la nueva normativa, que sin duda ha traído mejoras y avances muy importantes en materia de salud y seguridad en el trabajo en España, ha ido revelando también algunos de sus defectos y limitaciones (Luque, 2004:1-2; Boix, 2008:177). El énfasis de las empresas y servicios de prevención en un mero cumplimiento formal de las obligaciones establecidas lleva aparejado una excesiva burocratización en la aplicación de las normas y la denominada "prevención defensiva”, cuyos beneficios reales en términos de salud y seguridad de los trabajadores son cuanto menos dudosos. La prevención defensiva se ha asimilado con un sesgo jurídico de la prevención: empresas y servicios de prevención condicionan todas sus 
actuaciones a evitar la inspección y la sanción por parte de un sistema (Inspección de Trabajo) que, en el fondo, se percibe como bastante imprevisible y arbitrario. La norma jurídica no se interpreta a la luz del conocimiento técnico, de la experiencia propia o del sentido común, sino que invade todo el terreno, convirtiéndose en un fin cuando debería ser un medio; esto es, como si no existiera una prevención de riesgos como disciplina técnica, sino sólo como realidad jurídica. Ello sea quizás debido al hecho de que "nuestro marco jurídico actual, constituido en su esencia por la Ley de Prevención de Riesgos Laborales y sus reglamentos de desarrollo, es más el fruto obligado de nuestra pertenencia a la Unión Europea que el de un proceso interno de maduración en lo social, en lo político, en lo científico y en lo profesional" (Uberti y Rodrigo, 2006:118).

\section{LA ORGANIZACIÓN DE LA SALUD Y SEGURIDAD EN EL TRABAJO EN LAS EMPRESAS}

En la Ley de Prevención de Riesgos Laborales (31/1995) se ofrecen distintas modalidades para la organización de la prevención en las empresas. Sin embargo, en el Reglamento de los Servicios de Prevención (39/1997) se le otorga un protagonismo absoluto al servicio de prevención ajeno en clara e injustificada contraposición con lo que se postula en el artículo séptimo de la Directiva Marco. En este contexto, las Mutuas de Accidentes de Trabajo y Enfermedades Profesionales (MATEPs) han sido las principales beneficiarias del énfasis sobre la organización de la prevención en las empresas, contratándolas a través de servicios de prevención ajenos (actuales sociedades de prevención en las MATEPs).

Según datos de la Encuesta Nacional de Condiciones de Trabajo en España de 2009, un 73\% de las empresas se han decantado por contratar la prevención con entidades ajenas, imponiéndose progresivamente en el tiempo esta opción sobre el resto de modalidades que contempla la normativa. Consecuencia de ello, las opciones para planificar y gestionar la prevención con recursos propios de la empresa (apoyándose quizás en un mínimo asesoramiento externo) son prácticamente inexistentes, con la excepción de las compañías de mayor tamaño. La mayoría de empresas españolas se encuentran en los estratos más bajos según tamaño de empresa; sin embargo, también es cierto que una proporción importante de trabajadores en España están empleados en las empresas más grandes (Tabla 1).

La preferencia de las empresas por contratar los servicios de prevención a una entidad ajena ha sido reiteradamente referida como causante de la falta de integración de la prevención de riesgos laborales en las empresas, fenómeno que los expertos identifican como uno de los obstáculos principales para avanzar en materia de salud y seguridad en el trabajo en España (Velázquez, 2009:166). En efecto, si bien la obligación del empresario de "integrar" la prevención en su gestión ordinaria de la empresa se encuentra prevista tanto en la Ley de Prevención de Riesgos Laborales 
31/1995 como en la Directiva Marco 89/391/CEE y, en consecuencia, no se debería desdoblar la gestión de la producción y la gestión de la prevención, y la responsabilidad de la prevención sólo debería ser asumida por el propio empresario, sin posibilidad de delegarla en otros sujetos -ya sean trabajadores, servicios de prevención u otras empresas subcontratistas-, en realidad las prácticas de empresas, servicios de prevención y la propia administración distan mucho de favorecer dicha integración.

Tabla 1. Distribución de las empresas españolas según tamaño de la plantilla (Instituto Nacional de Estadística, 2011)

\begin{tabular}{|l|c|}
\cline { 2 - 2 } \multicolumn{1}{c|}{} & $\begin{array}{c}\text { Empresas } \\
\mathbf{n}(\%)\end{array}$ \\
\hline $1-5$ trabajadores & $1.161 .360(79,8)$ \\
\hline $6-49$ trabajadores & $269.034(18,5)$ \\
\hline $50-99$ trabajadores & $13.279(0,9)$ \\
\hline $100-499$ trabajadores & $9.873(0,7)$ \\
\hline 500 trabajadores y más & $1.709(0,1)$ \\
\hline
\end{tabular}

La actividad de los servicios de prevención en España ha sido objeto frecuente de escrutinio y crítica, y motivo también de iniciativas de profesionales, investigadores e instituciones con recomendaciones para mejorar su calidad y fomentar las buenas prácticas en los profesionales (Boix, 2003:7-14; Boix, 2011:177-178).

\section{ESTRUCTURA DE PARTICIPACIÓN EN LA PROTECCIÓN DE LA SALUD Y SEGURIDAD EN EL TRABAJO EN LAS EMPRESAS}

La Ley 31/1995 exige de forma explícita a los empresarios españoles, por primera vez en nuestra historia, la gestión de la salud y seguridad en el trabajo de forma sistemática, informada y participativa. Los lugares de trabajo con seis o más empleados deben elegir representantes de los trabajadores a través de los cuáles se canalice la participación de los trabajadores en las cuestiones que afecten a la prevención de riesgos laborales en la empresa. Son los llamados delegados de prevención. En la mayoría de los casos, estos delegados están vinculados a un sindicato y proceden de estructuras de participación ya existentes en la empresa (comités de empresa, delegados de personal). En su mayoría, también, dependen de las estructuras y recursos de apoyo de los dos sindicatos mayoritarios en España (CC.OO. y UGT). Así por ejemplo, en CC.OO. se ha organizado una amplia red de asesores en salud laboral con el objetivo principal de apoyar la actividad de sus delegados de prevención. Dicha red se dirige y coordina fundamentalmente desde el Instituto Sindical de Trabajo, Ambiente y Salud (ISTAS) de CC.OO (García et al, 2009:80; Narocki et al, 2011:3). 
En la Tabla 2 se describen las tareas y derechos de los Delegados de Prevención en España, tal y como vienen establecidas en la Ley 31/1995.

Tabla 2. Funciones y Derechos de los Delegados de Prevención en España (Ley 31/1995)

\section{FUNCIONES}

- $\quad$ Control del cumplimiento de la normativa sobre prevención de riesgos laborales (PRL).

- $\quad$ Promoción de la colaboración de los trabajadores en materia de PRL.

- Colaboración con los empresarios en procesos de decisión acerca de:

- Equipos de trabajo y condiciones ambientales.

- Organización de la actividad preventiva.

- Acciones en material de PRL.

- Procedimientos de información y formación delos trabajadores en material de PRL.

- Procedimientos de documentación en relación con los planes de prevención, la evaluación de riesgos laborales, la planificación de la PRL, la vigilancia de la salud de los trabajadores y las enfermedades y accidentes de trabajo.

- Elaboración de informes en respuesta a consultas de los empresarios.

- Respeto a la confidencialidad.

- Participación y desarrollo de las actividades llevadas a cabo por el Comité de Seguridad y Salud.

\section{DERECHOS}

- Ser consultados por los empleadores en las decisiones acerca de salud y seguridad en la empresa.

- Acompañar a los técnicos de PRL en las evaluaciones de riesgos.

- Acompañar a los inspectores de trabajo en sus visitas a las empresas por temas de PRL.

- Ser informados por los inspectores de trabajo de los resultados de sus visitas.

- Solicitar inspecciones a las autoridades laborales.

- Acceso libre a los informes y documentos de seguridad y salud disponibles en la empresa.

- Ser informados por los empleadores de los accidentes y enfermedades del trabajo que ocurran en la empresa.

- Ser informados por los empleadores sobre cualquier otro aspecto relacionado con la PRL.

- Visitar los puestos y lugares de trabajo para controlar las condiciones de trabajo.

- Hacer recomendaciones para la PRL al empleador y al Comité de Seguridad y Salud de la empresa.

- Recibir justificación por parte del empleador con respecto a las recomendaciones no implementadas.

- Parar la actividad laboral en caso de riesgo grave e inminente para la salud de los trabajadores.

- Recibir por parte del empleador la capacitación y recursos necesarios para el desarrollo de sus funciones.

- Especial consideración del tiempo de trabajo para la implementación de sus funciones.

- Garantía de sus condiciones de empleo y de su promoción profesional. 
Según la Encuesta Nacional de Gestión de la Seguridad y Salud en las Empresas de 2009 (Almodóvar y Pinilla, 2009:23), que incluye información con datos ponderados y representativos a nivel nacional, la cobertura de delegados de prevención en las empresas no es completa pero abarca una proporción relevante de centros. Esta cobertura se asocia con el tamaño de la empresa (Figura 1).

Figura 1. Empresas Españolas (\%) con Delegados de Prevención Constituidos

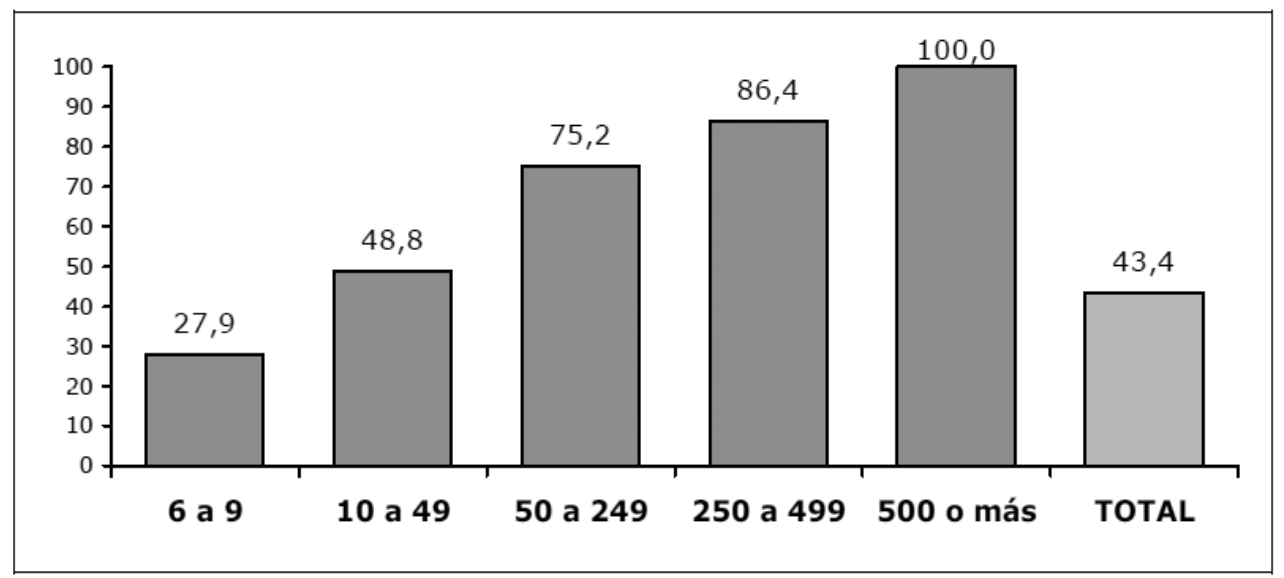

Fuente: Encuesta Nacional de Gestión de la Seguridad y Salud en las Empresas en España, 2009

La Ley 31/1995 establece también la constitución del Comité de Seguridad y Salud en todas las empresas con 50 trabajadores o más. El Comité de Seguridad y Salud es un órgano paritario formado por representantes de los trabajadores y de la dirección de la empresa con funciones de información y consulta sobre los aspectos relacionados con la salud laboral en los centros de trabajo. La disponibilidad de esta estructura de participación es muy alta en España: según la Encuesta Nacional de Condiciones de Trabajo de 2007 (Almodóvar y Pinilla, 2007:26-27), realizada sobre una muestra representativa de trabajadores ocupados de todas las actividades económicas en España, el 84\% de los centros de trabajo de 50 o más empleados cuentan con Comité de Seguridad y Salud, proporción que asciende al 92\% de los centros con 500 o más empleados. Las diferencias en la cobertura de los órganos y figuras de representación en materia de salud y seguridad en el trabajo en las empresas se observan no sólo en relación con el tamaño de los centros de trabajo, sino también en función del sector de actividad de las empresas, siendo estas estructuras y representantes más frecuentes en empresas del sector de la industria, seguidas por el sector servicios y la construcción. 
Desde la salud pública se ha reivindicado insistentemente el derecho a la participación ciudadana en las políticas que afecten a la protección de la salud, un elemento que la propia Organización Mundial de la Salud considera esencial para cuidar y mejorar la salud de la población (Segura, 2010:55). En muy pocos campos de acción de la salud pública se cuenta con estructuras de participación de ciudadanos sanos tan generalizadas, operativas y próximas a la población diana como en el ámbito de la salud laboral (Benavides y García, 2010:126). La presencia de delegados de prevención y de comités de seguridad y salud en un gran número de empresas españolas constituye una estructura única y efectiva para garantizar la participación de las personas en materia de salud. Esta estructura, como ha demostrado también la literatura científica es, además, efectiva para proteger y mejorar la salud de los trabajadores.

\section{IMPACTO DE LA PARTICIPACIÓN EN LA MEJORA Y PROTECCIÓN DE LA SALUD DE LOS TRABAJADORES: ALGUNAS EVIDENCIAS}

La efectividad de la participación en materia de salud y seguridad en el trabajo ha sido estudiada y evaluada por parte de la comunidad científica. Aunque este campo de investigación es todavía relativamente reducido, a continuación se comentan algunas de las evidencias disponibles.

\subsection{El "Efecto Sindicato" ("Union EFFEct")}

La agrupación de organizaciones sindicales Trade Union Congress (TUC) del Reino Unido defiende en un informe de 2011 (Trades Union Congress, 2011:3) lo que denomina como "efecto sindicato". En el informe se revisa la evidencia recogida en diferentes investigaciones a nivel internacional, que constata que en las empresas donde actúan representantes de los trabajadores en materia de salud laboral las condiciones de trabajo son mejores, se producen menos accidentes de trabajo y se cumple más la normativa. Según estos estudios, el "efecto sindicato" sería tanto resultado de las prácticas de los delegados de prevención en los centros de trabajo como de la existencia de organizaciones sindicales que los apoyan. Los autores de este informe concluían con la propuesta de una serie de medidas que potenciarían este impacto positivo en las empresas:

- Potenciar la figura del delegado territorial (o sectorial) que permitiría una mejor incorporación de las PYMEs en esta estructura de participación, incluyendo las subcontratas.

- Con el apoyo de la administración laboral, facilitar y potenciar para los representantes de los trabajadores mecanismos de advertencia para los empleadores que no cumplen con la normativa, incluyendo en su caso visitas de la inspección de trabajo. 
- Establecer mecanismos que fuercen a los empleadores a responder a los requerimientos para la prevención de riesgos laborales presentados por parte de los representantes de los trabajadores.

\subsection{El Proyecto EPSARE}

El proyecto EPSARE (Impacto de los delegados de prevención en la salud laboral en la Unión Europea) ha permitido recientemente realizar una valoración global de las condiciones y factores fundamentales que influyen en el impacto de los delegados de prevención en la salud laboral en el contexto europeo (Menéndez et al, 2008:5; Menéndez et al, 2009:1-32). En este proyecto se ha realizado una revisión exhaustiva de la literatura disponible, incluyendo también información de estudios epidemiológicos descriptivos mediante varias encuestas europeas y nacionales, y el conocimiento aportado por informantes clave como delegados de prevención, sindicalistas y expertos e investigadores en salud laboral. EPSARE tiene también como objetivos la mejora de la interacción entre sindicalistas, expertos y científicos, así como también facilitar el desarrollo de información e instrumentos prácticos preventivos para los delegados de prevención y sindicalistas europeos.

Entre las conclusiones del EPSARE se afirma que aunque el impacto de los representantes de seguridad y salud en el trabajo apenas se contempla en las agendas políticas y de investigación, el conocimiento disponible confirma que los sindicatos y los representantes en esta materia son un poderoso recurso para mejorar la salud de los trabajadores en la Unión Europea. La investigación llevada a cabo principalmente en países nórdicos europeos y en Norteamérica muestra que los lugares de trabajo donde existen delegados sindicales activos en esta materia son más seguros y presentan mejores indicadores de salud laboral.

\subsection{Encuesta Europea de Empresas sobre Riesgos Nuevos y Emergentes}

La Encuesta Europea de Empresas sobre Riesgos Nuevos y Emergentes (ESENER), encargada por la Agencia Europea para la Seguridad y Salud en el trabajo (EU-OSHA), se completó en junio de 2009 con unas 36.000 entrevistas telefónicas individuales en treinta y un países incluyendo a los veintisiete Estados miembros de la Unión Europea, dos países candidatos (Croacia y Turquía) y dos países de la Asociación Europea de Libre Comercio (Noruega y Suiza). Las entrevistas se realizaron a responsables encargados de la prevención de riesgos laborales y representantes de los trabajadores en esta materia. El objetivo principal era obtener información acerca de la gestión de los riesgos para la salud y la seguridad en sus respectivos lugares de trabajo, con especial atención a los riesgos psicosociales (Agencia Europea para la Seguridad y Salud en el Trabajo, online). Uno de los informes elaborados con los datos ESENER se centró en analizar los resultados en relación con aspectos de participación y consulta de los 
trabajadores en materia de seguridad y salud, investigando la relación entre la eficacia de las medidas de gestión y la participación de los representantes de los trabajadores en dichas medidas. De este análisis se obtuvieron las siguientes conclusiones:

- La representación de los trabajadores es más común en las grandes empresas, en el sector público, en organizaciones con una proporción de trabajadores de edad avanzada y en los lugares de trabajo donde la seguridad y salud y las opiniones de los trabajadores se perciben como elementos prioritarios.

- En los países escandinavos es más frecuente que en las empresas se cuente con representantes de los trabajadores, tanto a nivel general como en relación con la prevención de riesgos laborales, mientras que la proporción de empresas con estos representantes es menor en los países del sur de Europa.

- El compromiso de la dirección con la seguridad y la salud, en combinación con la representación de los trabajadores (particularmente con la representación en materia de seguridad y salud), se asocia positivamente con la existencia de medidas adecuadas para la gestión de la seguridad y salud en el trabajo, incluyendo la existencia de una política al respecto, la recogida rutinaria de datos sobre bajas por enfermedad y controles periódicos en el lugar de trabajo.

- Los trabajadores encuestados de empresas en las que existen tanto regímenes de representación de los trabajadores como un elevado grado de compromiso de la dirección con la seguridad y salud tienen casi diez veces más probabilidades de referir que su organización aplica una política de salud y seguridad documentada que los encuestados de lugares de trabajo en los que no hay representación de los trabajadores y el compromiso de la dirección con la salud y la seguridad es escaso.

- Es más probable que las medidas de gestión de la seguridad y salud en el trabajo se perciban como acciones eficaces en los lugares de trabajo en los que existe representación de los trabajadores y, en particular, cuando ésta se combina con un alto grado de compromiso de la dirección con la seguridad y la salud.

- En aquellas organizaciones que cuentan con representación de los trabajadores y que, además, dotan a esta representación de un contexto apropiado en el que actuar, es más probable que exista gestión efectiva de la seguridad y salud en el trabajo y que ésta sea más eficaz.

\subsection{Los Delegados de Prevención en España}

El Instituto Sindical de Trabajo, Ambiente y Salud de CC.OO. ha llevado a cabo una serie de encuestas y estudios con sus delegados de prevención en las empresas españolas. 
En el primer estudio (García et al, 2004:26-36, 46-57; García et al, 2005:139), realizado en 2003 mediante la aplicación de un cuestionario autocumplimentado entre los asistentes al I Encuentro Nacional de Delegados de Prevención, se observaba que las actividades más frecuentes de los delgados son la recogida de quejas y opiniones de los trabajadores así como la información y sensibilización de los mismos. En este mismo trabajo, los delegados señalaban como principales obstáculos para el desarrollo de sus funciones una mala actitud de los responsables de la empresa y la escasa presión por parte de la Inspección de Trabajo. Para conseguir reducir y eliminar riesgos laborales, los delegados pedían más formación, mejores cláusulas en los convenios e instrumentos para fomentar la participación de los trabajadores. En conclusión, esta primera encuesta parece indicar que los delegados centran su actividad y esfuerzos en torno a los trabajadores que representan. Sin embargo, los objetivos deseables en prevención de riesgos laborales parecen difícilmente alcanzables sin el necesario apoyo por parte de la empresa y de la administración.

Posteriormente, este estudio fue ampliado con una nueva investigación en la que se contempló la obtención tanto de información cuantitativa, a partir de la realización de una encuesta telefónica sobre una muestra representativa de los delegados de prevención a nivel nacional, como de información cualitativa, basada en la realización de grupos de discusión con delegados de prevención (Rodrigo y García, 2005:11-23). En esta segunda investigación, los delgados señalan que la formación de los trabajadores debe incluir no sólo la prevención de riesgos, sino el conocimiento de sus derechos de representación en materia de salud laboral y de las funciones de los delegados de prevención. Se observa también que la intervención de los delegados en la planificación, gestión y ejecución de la prevención en la empresa es más frecuente en las empresas mayores (>100 trabajadores), en las que los delegados de prevención son también en general más activos. Asimismo, la mayoría de delegados piensan que deben actuar en relación con los problemas medioambientales que genere la empresa, aunque también comparten la idea de que pueden influir poco o nada para evitar estos problemas y declaran carecer de formación suficiente sobre este aspecto.

El II Encuentro de Delegados de Prevención de CC.OO., celebrado en Madrid en octubre de 2009, se aprovechó de nuevo para pasar un cuestionario entre los asistentes. Se estima que en el encuentro participaron alrededor de 2.000 delegados y representantes de los trabajadores, de los que 1.229 completaron un cuestionario autocumplimentado con preguntas cerradas de opción múltiple. Los encuestados en su mayoría eran hombres (67\%), con edades comprendidas entre 36 y 55 años (76\%). Más de la mitad contaban con tres y más años de experiencia como delegados. El 71\% de los encuestados trabajaban en empresas con más de 100 trabajadores. El sector mayoritario de actividad de las empresas de los delegados encuestados era la industria manufacturera (28\%). Aunque en el encuentro había delegados de prácticamente todas las comunidades autónomas, la mayor representación procedía de Madrid (Roncal, 2010:13). 
En la siguiente tabla se describen las actividades que los delegados que participaron en este último estudio declaraban realizar con mayor y menor frecuencia en sus centros de trabajo.

Tabla 3. Actividades de Realizadas con Mayor y Menor Frecuencia por los Delegados de PREVENCIÓN

\section{Actividades}

n

$\%$ *

Actividades más frecuentes:

Atención de consultas de trabajadores

1.190

98,5

Dar información a los trabajadores

98,5

Pedir información a los trabajadores

95,1

Actividades menos frecuentes:

Propuesta de paralización de actividades por riesgo inminente

Coordinación con contratas/subcontratas

Declaración de Enfermedades Profesionales

* Porcentajes basados en el total excluyendo las no respuestas

Fuente: II Encuentro de Delegados de Prevención de CC.OO., Madrid 2009 (n=1.229)

Adicionalmente, los delegados perciben positivamente el impacto de su actividad en la empresa, como se deduce de los resultados presentados en la Tabla 4. Sin embargo, los delegados también califican en términos moderados su nivel de conocimiento en relación con sus funciones y actividades (sólo el 43\% los valoran como "altos o muy altos") y su satisfacción en relación con las actividades que desarrollan y los logros conseguidos (respectivamente, el 42\% y el 27\% declaran esta satisfacción como "alta o muy alta").

Tabla 4. Influencia de las Actividades Realizadas como Delegado de Prevención.

\begin{tabular}{lcc}
\hline & $\mathbf{n}$ & \%* \\
Influyeron en la mejora de condiciones de trabajo en la empresa & 1.058 & 90,4 \\
Influyeron en la eliminación o disminución de riesgos laborales en la empresa & 1.022 & 87,2 \\
Influyeron en la sensibilización de los trabajadores sobre los riesgos & 988 & 84,7 \\
Influyeron en la visualización de problemas ocultos o insuficientemente tratados & 927 & 80,2 \\
Influyeron en la mejora de las actividades realizadas por el servicio de prevención & 913 & 78,4 \\
\hline
\end{tabular}

* Porcentajes basados en el total excluyendo las no respuestas

Fuente: II Encuentro de Delegados de Prevención de CC.OO., Madrid 2009 (n=1.229) 


\section{CONCLUSIONES}

En este texto se han revisado evidencias y datos en relación con la participación de los trabajadores en la gestión de la prevención de riesgos laborales en las empresas, procedentes de España y de otros países de nuestro entorno.

En general, la evidencia internacional, aunque se considera todavía escasa, señala un efecto positivo de la participación de los trabajadores sobre las actividades y resultados en salud laboral de las empresas: la gestión de la salud y seguridad en el trabajo es más adecuada, las condiciones de trabajo son mejores y el impacto en la salud en términos de enfermedades y accidentes disminuye en las empresas que cuentan con mecanismos de participación de los trabajadores en esta materia, muy especialmente cuando se asocian a una adecuada implicación de los responsables y decisores de la empresa en los aspectos de prevención.

En comparación con otros países, en España contamos todavía con una corta experiencia en la incorporación de estructuras y representantes de los trabajadores con funciones específicas en materia de prevención de riesgos laborales; en concreto, los comités de seguridad y salud y los delegados de prevención en las empresas. Algunos informes y datos disponibles señalan que, aunque la cobertura es elevada, todavía no es completa, y también que la presencia de estos recursos no se distribuye de forma homogénea entre las empresas españolas: son más frecuentes en las empresas más grandes y en los sectores de industria y servicios. Los delegados de prevención son muy activos en sus lugares de trabajo, aunque las actividades más frecuentes se relacionan con la formación e información de los trabajadores, teniendo menos presencia en los procesos de planificación de la prevención y en la acción preventiva que puedan desarrollarse en las empresas. En general, los delegados reclaman mayor implicación en salud y seguridad en el trabajo tanto por parte de los empresarios como de la administración.

Algunos informes europeos señalan la importancia de la cobertura hacia las empresas más pequeñas, poco o nada sindicalizadas, en las que la figura del delegado territorial y/o sectorial podría paliar las deficiencias. En España son casi inexistentes las experiencias en este sentido. También se insiste en la necesidad de mecanismos que induzcan a las empresas a implicarse en mayor medida en sus obligaciones en materia de salud laboral, en línea con la insistentemente reclamada integración de la prevención en todas las actividades de la empresa (en oposición a la habitual externalización de las actividades preventivas), así como de una mayor presión por parte de la administración en apoyo a los delegados de prevención y en cumplimiento de la normativa vigente por parte de las empresas. 


\section{BIBLIOGRAFIA}

Agencia Europea para la Seguridad y Salud en el Trabajo. Encuesta europea de empresas sobre riesgos nuevos y emergentes (ESENER); 2010 [consultado 18 mayo 2013]. Disponible en:

https://osha.europa.eu/es/publications/reports/es_esener1-summary.pdf/view

Agencia Europea para la Seguridad y Salud en el Trabajo. Conocer la gestión en el lugar de trabajo de la seguridad y salud, los riesgos psicosociales y la participación de los trabajadores, con la ayuda de ESENER; 2012 [consultado 18 mayo 2013]. Disponible en:

https://osha.europa.eu/es/publications/reports/esener-summary

Almodóvar, A, y Pinilla, F.J. (coord.) (2007): VI Encuesta Nacional de Condiciones de Trabajo, Madrid, Instituto Nacional de Seguridad e Higiene en el Trabajo.

Almodovar, A, y Pinilla, F.J. (coord.) (2009): Encuesta Nacional de Gestión de la Seguridad y Salud en las Empresas, Madrid, Instituto Nacional de Seguridad e Higiene en el Trabajo.

Benavides, F.G. y García, A.M. (2010): "Participación ciudadana en salud: el caso de la salud laboral”, Gestión Clinica y Sanitaria, 12(4), pp. 126.

Bernabeu, J.; Perdiguero, E. y Zaragoza, P. (2000). "Desarrollo histórico de la salud laboral”, F.G. Benavides, C. Ruiz-Frutos y A.M. García (edit.), Salud laboral: conceptos y técnicas para la prevención de riesgos laborales, $2^{\mathrm{a}}$ edición. Barcelona: Editorial Masson, pp. 71-81.

Boix, P. (edit.) (2003), Hacer lo que hay que bacer y bacerlo bien. III Foro ISTAS de Salud Laboral, Madrid, Instituto Sindical de Trabajo, Ambiente y Salud.

Boix, P.; Rodrigo, F. y García, A.M. (2008), Informe sobre la calidad de los servicios de prevención en España, Madrid, Instituto Sindical de Trabajo, Ambiente y Salud.

Boix, P. y Rodríguez de Prada, A. (2011), "Buena práctica profesional y calidad de la prevención: una iniciativa de transferencia impulsada por el Instituto Nacional de Seguridad e Higiene en el Trabajo", Arch Prev Riesgos Labor, 14, pp.177-8.

García, A.M.; Rodrigo, F.; Dudzinski, I. y López-Jacob, M.J. (2004), Análisis de las tareas y percepciones de los delegados y delegadas de prevención en España, Madrid, Instituto Sindical de Trabajo, Ambiente y Salud.

García, A.M.; Gadea, R. y Rodrigo, F. (2005), "Prevención de riesgos laborales en las empresas: percepciones de los delegados de prevención”, Arch Prev Riesgos Labor, 8(4), pp.139-146. 
García, A.M.; López-Jacob, M.J.; Dudzinski, I.; Gadea, R. y Rodrigo, F. (2009), Characteristics, activities and perceptions of Spanish safety representatives, D. Walters \& T. Nichols (edit.), Workplace health and safety. International perspectives on worker representation, Hampshire, Palgrave MacMillan, pp. 73-89.

García, A.M. (2012). Proyecto docente. Valencia: Concurso de acceso para la plaza código 61/2011 del cuerpo docente de Catedrático de Universidad, área de conocimiento Medicina Preventiva y Salud Pública en el Departamento de Medicina Preventiva y Salud Pública, Ciencias de la Alimentación, Toxicología y Medicina Legal de la Universitat de València (BOE de 20 de diciembre de 2011) [mimeo].

Luque, M. (2004), "La reforma de la Ley de Prevención de Riesgos Laborales: una primera valoración”, Arch Prev Riesgos Labor, 7 (1), pp.1-2

Menéndez, M.; Benach, J. y Vogel, L. (2008), "El impacto de los delegados de prevención en la salud laboral: el proyecto EPSARE", Arch Prev Riesgos Labor, 11 (1), pp.5-7

Menéndez, M.; Benach, J. y Vogel, L. (coord.) (2009), The impact of safety representatives on occupational health. A European perspective, Bruselas, European Trade Union Institute (ETUI), report 107.

Narocki, C.; López-Jacob, M.J.; Canaleta, E.; Morán Barrero, P. y Briso-Montiano, P. (2011), Impacto del asesoramiento sindical en salud laboral. Madrid, Instituto Sindical de Trabajo, Ambiente y Salud.

Rodrigo, F. y García, A.M. (2005), Los delegados de prevención en España: estado de situación, Madrid, Instituto Sindical de Trabajo, Ambiente y Salud.

Roncal, A. (2010). Prevención de riesgos laborales en las empresas: análisis de las actividades y percepciones de los delegados de prevención en España. Barcelona: Proyecto Final del Curso Superior de Medicina del Trabajo de la Unidad Docente de Medicina del Trabajo "Mateu Orfila" de la Universitat Pompeu Fabra de Barcelona [mimeo]

Segura, A. (2010), "La participación ciudadana, la sanidad y la salud", Gest Clín Sanit, 12 , pp. $55-62$.

Trades Union Congress (2011), How unions make a difference to bealth and safety: the unión effect, Londres, OSD/Health and Safety.

Uberti-Bona, V. y Rodrigo Cencillo, F. (2006), "Sobre sistemas de inspección y sistemas de prevención", Arch Prev Riesgos Labor, 9 (3), pp.117-20.

Velázquez, M. (2009), "La integración de la prevención de riesgos laborales en la empresa", Arch Prev Riesgos Labor, 12 (4), pp.165-9. 
